\title{
A discrete-time mathematical model of stage-structured mosquito populations
}

\author{
Yanyuan Xing ${ }^{1,2}$, Jian Liu' ${ }^{1}$ and Zhiming Guo ${ }^{1 *}$
}

"Correspondence:

guozm@gzhu.edu.cn

'School of Mathematics and Information Sciences, Guangzhou University, Guangzhou, P.R. China Full list of author information is

available at the end of the article

\section{Springer}

\begin{abstract}
This paper proposes a system of difference equations to model mosquito population. In this study, we develop and analyze the stage-structured models which consist of four distinct mosquito metamorphic stages: eggs, larvae, pupae, and adults. First, a model with constant birth rate is studied, and the inherent net reproduction number $\Re_{0}$ of the model is derived. If $\Re_{0}<1$, the extinction equilibrium is globally asymptotically stable. If $\Re_{0}>1$, there exists a unique positive equilibrium which is uniformly persistent. When breeding is seasonal for a special case, it indicates that a unique globally asymptotically stable periodic solution is admitted when the net reproductive number is larger than one. When this value is less than one, the mosquito population goes to extinction. Finally, numerical simulations to demonstrate our findings and brief discussion are also provided.
\end{abstract}

Keywords: Global stability; Net reproductive number; Extinction; Persistence; Mosquito population dynamics

\section{Introduction}

The Asian tiger mosquito or forest day mosquito (Aedes albopictus), from the mosquito family Culicidae, is characterized by its black and white striped legs, small black and white body. It is native in the tropical and subtropical areas of Southeast Asia. Over the past few decades, this species has invaded many countries around the world through the increasing transport of goods and international travel [1]. It is a competent vector for more than 25 arboviruses, including dengue, Zika [2] and chikungunya [1], which was first reported in the Reunion Island in March 2005. For controlling the mosquito-borne diseases, the most traditional measures are to directly kill mosquitoes by spraying insecticides, reducing the source of larvae, and eliminating mosquitoes. On the one hand, although the current vector control strategies, including insecticide spraying and community-based source reduction, have taken some effect in a short time period, innovative prevention and control methods with long-term effect are still needed to control mosquito-borne disease. On the other hand, due to the lack of commercially available vaccines for dengue, Zika, and chikungunya, current mosquito-borne disease control methods focus on suppressing the prevalence of vectors and their capacity to transmit viruses [3, 4]. Developing tools to better understand the dynamics of mosquito populations and control them is a critical area of research.

(c) The Author(s) 2019. This article is licensed under a Creative Commons Attribution 4.0 International License, which permits use sharing, adaptation, distribution and reproduction in any medium or format, as long as you give appropriate credit to the original author(s) and the source, provide a link to the Creative Commons licence, and indicate if changes were made. The images or other third party material in this article are included in the article's Creative Commons licence, unless indicated otherwise in a credit line to the material. If material is not included in the article's Creative Commons licence and your intended use is not permitted by statutory regulation or exceeds the permitted use, you will need to obtain permission directly from the copyright holder. To view a copy of this licence, visit http://creativecommons.org/licenses/by/4.0/ 
The complete metamorphosis of the mosquito goes through four different stages of life cycle development, starting with the egg and hatching into larvae within a week under the right conditions. Larvae will use their tubes to breathe air by poking above the surface of the water and eat a bit of floating organic matter. Larvae molt four times totally when they grow and after the fourth molt, they are called pupae. Pupae also live near the surface of water and breathe through two horn-like tubes on their back, but pupae do not eat. When the skin splits after a few days from a pupa, an adult mosquito emerges [5]. Female mosquitoes then feed on human or animal blood to provide protein for their eggs. After biting, female mosquitoes rest while their eggs develop. Once eggs are fully developed, the females oviposit and then proceed to finding another blood meal to complete the mosquito feeding cycle [6]. The adults live for only a few weeks, and a full life-cycle of a mosquito takes about a month.

To have more realistic modelling of mosquitoes, we need to study stage structure, because the different stages respond to environment differently and regulate the population differently. Comparing the homogeneous stage-structured models, the latter model is more reasonable and realistic. Our goal is, after having a fundamental understanding of the dynamics for the mosquitoes, to have the mosquito models incorporated into disease transmission models for the mosquito-borne diseases [7]. There have been many elaborate works for the dynamical behavior of the transmission of mosquito-borne diseases [8-10], but many of these models do not take into account the metamorphic structure differences of mosquito populations. As we know, individuals differ in size or developmental stage, they also differ in their vital rates. Recently, continuous-time dynamical systems of stage-structured mosquito populations have been studied [9, 11]. However, since the experimental data of the detection of mosquito population in the field are discrete, we ought to establish a discrete model no matter considering the research background or the rationality of the model establishment. In this paper, our model is of discrete time and based on difference equations. The discrete model is referred to what we may call a discrete domain. The structure is indeed decomposed into parts called elements, and the set of such parts is what we call the discrete domain to which the discrete model is referred. Moreover, we consider stage-structured mosquito populations without distinguishing male and female individuals and assume that the mosquito dynamics follow the nonlinearity of Beverton-Holt type. Also, it should be noticed that interspecific competition and predation are rather rare events in mosquito populations. In this context, matrix models can describe population changes from one time step to the next. Commonly, it takes the form: $x(t+1)=B(x(t)) x(t)$, where $x(t)$ is a vector of counts or densities at each stage at time $t$, and $B(x(t))=\left(b_{i j}\right)$ is a standard density-dependent population projection matrix detailing survival, maturation, and reproduction of existing individuals.

Recently, Lu and Li [12] studied the dynamics for mosquito population which included the discrete stage-structured model and formulated the following model:

$$
\left\{\begin{array}{l}
E(t+1)=b_{0} A(t), \\
L(t+1)=s_{e}(L(t)) E(t), \\
P(t+1)=s_{l}(L(t)) L(t), \\
A(t+1)=s_{p} P(t)+s_{a}(A(t)) A(t),
\end{array}\right.
$$


where the mosquitoes are divided into four stages: eggs, larvae, pupae, and adults. $E(t)$, $L(t), P(t), A(t)$ denote the numbers of mosquitoes in every stage, respectively, at time $t$. The remaining coefficients are denoted as follows:

- $b, s_{0}$ : rate of oviposition and survival probability;

- $s_{e}$ : hatching rate from eggs to larvae;

- $s_{l}$ : pupation rate from larvae to pupae;

- $s_{p}, s_{a}$ : emergence rate from pupae to adults and density-dependent survival function of adults.

In the process of mathematical modeling, the inhibition of larva density on egg hatching is considered. They assume the surviving-adjusted hatching rate, denoted by $s_{e}=s_{e}(L)$, to be a function of larvae $L$. These rates have the Beverton-Holt or Ricker-type form. It was shown by the analytical results that when the inherent net reproductive number is less than one, the trivial fixed point of system (1.1) is globally asymptotically stable, and is unstable if it is larger than one. However, for the unique positive fixed point, they only showed its local asymptotic stability under some conditions. Later, in 2017, Tian and Ruan [13] studied a free boundary problem for Aedes aegypti mosquito invasion, including the stage-structured mosquito population. Recently, Huang and Hu [14] developed a stagestructured model of delay differential equations that combines larval density-dependent competition with diapause eggs in order to compare the efficiency of different suppression strategies.

Based on an extensive review of current knowledge on mosquito biology, we estimate the seasonal oviposition rate that changes with the environmental conditions. Egg is treated by dividing one time unit into favorable (rainy season) and unfavorable (dry season) seasons during which adults will either not reproduce, or produce eggs that do not hatch. These lead us to considering the following discrete-time mathematical model to describe the dynamics of Aedes aegypti mosquito population, based on systems of difference equations:

$$
\left\{\begin{array}{l}
E(t+1)=b(t) A(t)+\left(1-\gamma_{1}\right) s_{1}(E(t)) E(t), \\
L(t+1)=\gamma_{1} s_{1}(E(t)) E(t)+\left(1-\gamma_{2}\right) s_{2}(L(t)) L(t), \\
P(t+1)=\gamma_{2} s_{2}(L(t)) L(t)+\left(1-\gamma_{3}\right) s_{3} P(t), \\
A(t+1)=\gamma_{3} s_{3} P(t)+s_{4}(A(t)) A(t), \\
(E(0), L(0), P(0), A(0)) \in \mathbb{R}_{+}^{4} \backslash(0,0,0,0) .
\end{array}\right.
$$

The state variables $E(t), L(t), P(t)$, and $A(t)$ represent the numbers of eggs, larvae, pupae, adults, respectively, at time $t$. The parameter $b(t)$ is the average number of eggs produced per female adult in one time unit, while $\gamma_{i}(i=1,2,3)$ represents the rate (in one time unit) of eggs that hatch into larvae, larvae develop into pupae, and pupae that become adult mosquitoes, respectively. Hence, we assume $0<\gamma_{i} \leq 1$ for $i=1,2,3$. The function $s_{i}(i=1,2,4)$ is the nonlinear survivorship rate of stage $i$. Since the pupae do not eat, we shall assume the survivorship rate from pupae to adults to be constant denoted by $s_{3}$, and $0<s_{3}<1$. Specially, interspecific competition and predation are rather rare events in the population dynamics of mosquitoes. Thus, we also assume that each stage of population only competes with themselves and are mutually independent. While only female mosquitoes are involved in the transmission of vector-borne diseases, this model ignores 
males. Furthermore, the mosquito survival rate in each stage $s_{i}$ has the following properties:

$$
\begin{gathered}
\left(H_{1}\right) \quad s_{i}(x) \in C^{1}[0, \infty), \quad s_{i}(0)=a_{i}, \quad 0<a_{i}<1, \quad s_{i}^{\prime}(x)<0, \quad \frac{d\left(s_{i}(x) x\right)}{d x}>0, \\
\lim _{x \rightarrow \infty} s_{i}(x)=0, \quad \lim _{x \rightarrow \infty} s_{i}(x) x=\widehat{a_{i}<\infty} \quad \text { for } i=1,2,4 .
\end{gathered}
$$

Clearly, $\left(H_{1}\right)$ is satisfied by the Beverton-Holt dynamics given by $s_{i}(x)=\alpha_{i} k_{i} /\left(k_{i}+\alpha_{i} x\right)$ for $i=1,2,4$. It represents that the surviving rates of hatching, pupation, and adult stage are all decreasing, but the total numbers of eggs, pupae, and adults are increasing and saturating to fixed numbers as the number of individuals increases [12]. Here, we are mainly interested in analysing model (1.2) for two cases. In the first case, we assume that $b(t)=b$, independent of $t$, is a positive constant, while in the second case, we assume that $b(t)$ is periodic with period 2. In this paper, our aim is to investigate the dynamical properties of a discrete stage-structured mosquito population with stage specific competition.

The remainder of this paper is organized as follows. In Sect. 2, we analyze global dynamical properties of the difference equation model when breeding is a constant, including basic reproductive number, existence of equilibria, stability of mosquito-free equilibrium $E_{0}$, as well as persistence of positive equilibrium $E_{1}$. In Sect. 3 , we study a special case when breeding is seasonal with period-2 birth rates and provide the conditions on the inherent net reproductive number that lead to the population reaching a globally asymptotically stable two-cycle or going to extinction. In Sect. 4, some numerical simulations are presented to interpret the behaviour of the model, and we explain our main results biologically. Meanwhile, we show numerically for the case where density-dependent vital rate has the Ricker-type form, which reveals much more complex dynamics. A short discussion on research results is also given in this section.

\section{Constant birth}

In this section, we consider system (1.2) where breeding is continuous. That is, for the remainder of this section, we assume that $b(t)=b$ is a positive constant. Thus, system (1.2) can be written as the following difference equation:

$$
x(t+1)=B(x(t)) x(t) .
$$

Here, $x(t)=(E(t), L(t), P(t), A(t))^{T}$, and the projection matrix $B$ that maps the density at time $t$ to the density at time $t+1$ has the form

$$
B(x)=\left(\begin{array}{cccc}
\left(1-\gamma_{1}\right) s_{1}(E) & 0 & 0 & b \\
\gamma_{1} s_{1}(E) & \left(1-\gamma_{2}\right) s_{2}(L) & 0 & 0 \\
0 & \gamma_{2} s_{2}(L) & \left(1-\gamma_{3}\right) s_{3} & 0 \\
0 & 0 & \gamma_{3} s_{3} & s_{4}(A)
\end{array}\right) .
$$

This allows us to determine the steady state values by solving the system of equations

$$
x=B(x) x .
$$

In population dynamic applications, we are interested in solutions with non-negative components $E(t) \geq 0, L(t) \geq 0, P(t) \geq 0, A(t) \geq 0$. Let $\mathbb{R}_{+}^{4} \doteq(0,+\infty) \times(0,+\infty) \times(0,+\infty) \times$ 
$(0,+\infty)$. It must be pointed out that the solution of system (1.2) remains non-negative. System (1.2) will always have a trivial steady state $E_{0}=(0,0,0,0)$, where all four of the population classes are zero. From $\left(H_{1}\right)$, we obtain that system (1.2) or (2.1) has the property: if $x \leq y, B(x) \geq B(y)$, where vector and matrix inequalities hold componentwise.

We shall use the techniques in [15-17] to find the net reproductive number $\Re_{0}$ of the population. Notice that the inherent projection matrix is $B(0)=G+T(0)$, where the transition matrix $T(0)$ is

$$
T(0)=\left(\begin{array}{cccc}
\left(1-\gamma_{1}\right) a_{1} & 0 & 0 & 0 \\
\gamma_{1} a_{1} & \left(1-\gamma_{2}\right) a_{2} & 0 & 0 \\
0 & \gamma_{2} a_{2} & \left(1-\gamma_{3}\right) s_{3} & 0 \\
0 & 0 & \gamma_{3} s_{3} & a_{4}
\end{array}\right),
$$

and the fertility matrix $G$ is

$$
G=\left(\begin{array}{cccc}
0 & 0 & 0 & b \\
0 & 0 & 0 & 0 \\
0 & 0 & 0 & 0 \\
0 & 0 & 0 & 0
\end{array}\right)
$$

Thus, the net reproductive number is the positive, simple, and strictly dominant eigenvalue of the matrix $G(I-T(0))^{-1}$. Through direct calculations, we know that

$$
\begin{aligned}
(I- & T(0))^{-1} \\
= & \frac{1}{\eta_{1} \eta_{2} \eta_{3}\left(1-a_{4}\right)} \\
& \times\left(\begin{array}{cccc}
\eta_{2} \eta_{3}\left(1-a_{4}\right) & 0 & 0 & 0 \\
\gamma_{1} a_{1} \eta_{3}\left(1-a_{4}\right) & \eta_{1} \eta_{3}\left(1-a_{4}\right) & 0 & 0 \\
\gamma_{1} \gamma_{2} a_{1} a_{2}\left(1-a_{4}\right) & \eta_{1} \gamma_{2} a_{2}\left(1-a_{4}\right) & \eta_{1} \eta_{2}\left(1-a_{4}\right) & \eta_{1} \eta_{2}\left(1-a_{4}\right) \\
\gamma_{1} \gamma_{2} \gamma_{3} a_{1} a_{2} s_{3} & \eta_{1} \gamma_{2} \gamma_{3} a_{2} s_{3} & \gamma_{3} s_{3} \eta_{1} \eta_{2} & \eta_{1} \eta_{2} \eta_{3}
\end{array}\right),
\end{aligned}
$$

where $\eta_{1}=1-\left(1-\gamma_{1}\right) a_{1}, \eta_{2}=1-\left(1-\gamma_{2}\right) a_{2}, \eta_{3}=1-\left(1-\gamma_{3}\right) s_{3}$. Thus,

$$
G(I-T(0))^{-1}=\frac{b \gamma_{1} \gamma_{2} \gamma_{3} a_{1} a_{2} s_{3}}{\eta_{1} \eta_{2} \eta_{3}\left(1-a_{4}\right)} .
$$

It is easily seen that

$$
\Re_{0}\left(\gamma_{1}, \gamma_{2}, \gamma_{3}\right)=\frac{b \gamma_{1} \gamma_{2} \gamma_{3} a_{1} a_{2} s_{3}}{\left(1-\left(1-\gamma_{1}\right) a_{1}\right)\left(1-\left(1-\gamma_{2}\right) a_{2}\right)\left(1-\left(1-\gamma_{3}\right) s_{3}\right)\left(1-a_{4}\right)} .
$$

It is worth noting that we use the notation $\Re_{0}\left(\gamma_{1}, \gamma_{2}, \gamma_{3}\right)$ to indicate the dependency of $\Re_{0}$ on $\gamma_{1}, \gamma_{2}$, and $\gamma_{3}$. Furthermore, if system (1.2) has a nontrivial interior steady state $E_{1}=(\widetilde{E}, \widetilde{L}, \widetilde{P}, \widetilde{A})$, where all stages have positive density, then $\widetilde{E}$-component of a nontrivial steady state must satisfy

$$
1=\left(1-\gamma_{1}\right) s_{1}(\widetilde{E})+\frac{b \gamma_{1} \gamma_{2} \gamma_{3} s_{1}(\widetilde{E}) s_{2}(\widetilde{L}) s_{3}}{\left(1-\left(1-\gamma_{2}\right) s_{2}(\widetilde{L})\right)\left(1-\left(1-\gamma_{3}\right) s_{3}\right)\left(1-s_{4}(\widetilde{A})\right)} \equiv F(\widetilde{E}),
$$


where

$$
\begin{aligned}
& \widetilde{L}=\frac{1}{1-\left(1-\gamma_{2}\right) s_{2}(\widetilde{L})} \gamma_{1} s_{1}(\widetilde{E}) \widetilde{E} \\
& \widetilde{P}=\frac{\gamma_{2} s_{2}(\widetilde{L}) \widetilde{L}}{1-\left(1-\gamma_{3}\right) s_{3}}=\frac{\gamma_{1} \gamma_{2} s_{1}(\widetilde{E}) s_{2}(\widetilde{L}) \widetilde{E}}{\left(1-\left(1-\gamma_{2}\right) s_{2}(\widetilde{L})\right)\left(1-\left(1-\gamma_{3}\right) s_{3}\right)}, \\
& \widetilde{A}=\frac{1}{1-s_{4}(\widetilde{A})} \gamma_{3} s_{3} \widetilde{P}=\frac{\gamma_{1} \gamma_{2} \gamma_{3} s_{1}(\widetilde{E}) s_{2}(\widetilde{L}) s_{3} \widetilde{E}}{\left(1-\left(1-\gamma_{2}\right) s_{2}(\widetilde{L})\right)\left(1-\left(1-\gamma_{3}\right) s_{3}\right)\left(1-s_{4}(\widetilde{A})\right)} .
\end{aligned}
$$

Since $F^{\prime}(\widetilde{E})<0$ and $\lim _{\widetilde{E} \rightarrow \infty} F(\widetilde{E})=0$, we see that if

$$
F(0)=\left(1-\gamma_{1}\right) a_{1}+\frac{b \gamma_{1} \gamma_{2} \gamma_{3} a_{1} a_{2} s_{3}}{\left(1-\left(1-\gamma_{2}\right) a_{2}\right)\left(1-\left(1-\gamma_{3}\right) s_{3}\right)\left(1-a_{4}\right)}>1
$$

which is equivalent to $\Re_{0}\left(\gamma_{1}, \gamma_{2}, \gamma_{3}\right)>1$, then system (1.2) has a nontrivial unique interior steady state $E_{1}=(\widetilde{E}, \widetilde{L}, \widetilde{P}, \widetilde{A})$. Furthermore, we shall show that system (1.2) is point dissipative. In fact, with assumption $\left(H_{1}\right)$, we obtain

$$
P(t+1)=\gamma_{2} s_{2}(L(t)) L(t)+\left(1-\gamma_{3}\right) s_{3} P(t) \leq \gamma_{2} \widehat{a_{2}}+s_{3} P(t) .
$$

Let $\overline{P(t+1)}$ satisfy the recursion $\overline{P(t+1)}=\gamma_{2} \widehat{a_{2}}+s_{3} \overline{P(t)}$. Then it is easy to see that $\overline{P(t+1)}=$ $\gamma_{2} \widehat{a_{2}} \sum_{j=0}^{t-1} s_{3}^{j}+s_{3}^{t} \overline{P(0)}$ and $\overline{P(t)}$ converges at $t \rightarrow \infty$. Since $0 \leq P(t) \leq \overline{P(t)}, P(t)$ is bounded as $t \rightarrow \infty$. Assume that $P(t) \leq M, \forall t=0,1,2, \ldots$. Then, it follows from system (1.2) that

$$
\begin{aligned}
& L(t) \leq \gamma_{1} \widehat{a_{1}}+\left(1-\gamma_{2}\right) \widehat{a_{2}}, \quad \forall t \geq 0, \\
& A(t) \leq \widehat{a_{4}}+\gamma_{3} s_{3} M:=\delta_{1}, \quad \forall t=1,2, \ldots, \\
& E(t) \leq b\left(\widehat{a_{4}}+\gamma_{3} s_{3} M\right)+\left(1-\gamma_{1}\right) \widehat{a_{1}}:=\delta_{2}, \quad \forall t=2,3, \ldots .
\end{aligned}
$$

Upon the positivity and boundedness of solutions for model (1.2), we claim the following result.

Lemma 2.1 Let $(E(t), L(t), P(t), A(t))$ be the solution of model (1.2). Then the positive octant $\{(E(t)>0, L(t)>0, P(t)>0, A(t)>0)\}$ is invariant, the sequence $(E(t), L(t), P(t), A(t)), t \geq 0$ is ultimately bounded for as $t \rightarrow \infty$. That is to say, there is a compact set $\Gamma \in \mathbb{R}_{+}^{4}$ such that every forward solution sequence of (1.2) enters $\Gamma$ in at most two time steps, and remain in $\Gamma$ forever after.

Proof The first part is obvious. As for the boundedness of solutions to model (1.2), we denote

$$
\begin{aligned}
\Gamma= & \left\{(E, L, P, M) \in \mathbb{R}_{+}^{4}: E \in\left[0, \delta_{2}\right], L \in\left[0, \gamma_{1} \widehat{a_{1}}+\left(1-\gamma_{2}\right) \widehat{a_{2}}\right],\right. \\
& \left.P \in[0, M], A \in\left[0, \delta_{1}\right]\right\} .
\end{aligned}
$$

This implies that the compact set $\Gamma$ is positively invariant and all the solutions are nonnegative and ultimately bounded. 
The following results can be verified by direct calculations.

(i) If $\Re_{0}\left(\gamma_{1}, \gamma_{2}, \gamma_{3}\right)<1$, then (1.2) always has a unique extinction equilibrium $E_{0}=(0,0,0,0)$.

(ii) If $\Re_{0}\left(\gamma_{1}, \gamma_{2}, \gamma_{3}\right)>1$, then (1.2) has a nontrivial equilibria, denoted by $E_{1}=(\widetilde{E}, \widetilde{L}, \widetilde{P}, \widetilde{A})$.

Thus, $\Re_{0}\left(\gamma_{1}, \gamma_{2}, \gamma_{3}\right)$, which indicates the average number of offspring produced per individual, plays a key role in determining the existence and stability of equilibria of model (1.2). Now, we prove the following stability result for (1.2). Notice that an equilibrium is globally asymptotically stable on $\mathbb{R}_{+}^{4}$ if it is locally asymptotically stable on $\mathbb{R}_{+}^{4}$ and if $(E(0), L(0), P(0), A(0)) \in \mathbb{R}_{+}^{4}$ implies that $(E(t), L(t), P(t), A(t))$ tends to the equilibrium as $t \rightarrow \infty$.

Theorem 2.1 If $\Re_{0}\left(\gamma_{1}, \gamma_{2}, \gamma_{3}\right)<1$, then the extinction equilibrium $E_{0}$ of model (1.2) is globally asymptotically stable. Moreover, $E_{0}$ is unstable if $\Re_{0}\left(\gamma_{1}, \gamma_{2}, \gamma_{3}\right)>1$.

Proof The proof is similar to that of [18-20] with some minor modifications. Since $\Re_{0}\left(\gamma_{1}, \gamma_{2}, \gamma_{3}\right)<1$, system (1.2) only has a trivial steady state $E_{0}=(0,0,0,0)$. Define the map $P: \mathbb{R}_{+}^{4} \rightarrow \mathbb{R}_{+}^{4}$ for the right-hand side of system (1.2). To calculate the stability of $E_{0}$, we need to linearize model (1.2) about steady state $E_{0}$ and evaluate the resulting Jacobian matrix

$$
J P\left(E_{0}\right)=\left(\begin{array}{cccc}
\left(1-\gamma_{1}\right) a_{1} & 0 & 0 & b \\
\gamma_{1} a_{1} & \left(1-\gamma_{2}\right) a_{2} & 0 & 0 \\
0 & \gamma_{2} a_{2} & \left(1-\gamma_{3}\right) a_{3} & 0 \\
0 & 0 & \gamma_{3} a_{3} & a_{4}
\end{array}\right) .
$$

Then the eigenvalues of $J P\left(E_{0}\right)$ have magnitude less than one, and hence, equilibrium $E_{0}$ is locally asymptotically stable.

We next establish global asymptotic stability of $E_{0}$. Note that the inherent projection matrix $B(0)$ of system (1.2) is non-negative, irreducible, and primitive. It has positive, simple, and strictly dominant eigenvalues $r$. Moreover, since $\Re_{0}\left(\gamma_{1}, \gamma_{2}, \gamma_{3}\right)<1$, it follows from [16] that $r<1$ and $\lim _{t \rightarrow \infty} B^{t}(0)=0$. Thus, for any $x(0)$, we have $0 \leq x(1)=B(x(0)) x(0) \leq$ $B(0) x(0)$, and repeating this we get that $0 \leq x(t) \leq B^{t}(0) x(0) \rightarrow 0$ as $t \rightarrow \infty$. Hence, $E_{0}$ is globally asymptotically stable. That is to say, if the extinction steady state is stable, the mosquito population can not persist.

In addition, when $\Re_{0}\left(\gamma_{1}, \gamma_{2}, \gamma_{3}\right)>1$, it follows from Theorem 1.1.3 in [16] that $B(0)$ has a positive strictly dominant eigenvalue greater than one. That is, the linearization of (1.2) at $E_{0}$ has a positive eigenvalue greater than one, which means trivial fixed point $E_{0}$ is unstable.

Theorem 2.2 If $\Re_{0}\left(\gamma_{1}, \gamma_{2}, \gamma_{3}\right)>1$, then system (1.2) is uniformly persistent.

Proof Assume $\Re_{0}\left(\gamma_{1}, \gamma_{2}, \gamma_{3}\right)>1$, let $f$ be the map on the right-hand side of $(1.2)$ from $\mathbb{R}_{+}^{4}$ to $\mathbb{R}_{+}^{4}$, and $D$ denotes the boundary of $\Gamma$ defined in (2.5). Since int $\mathbb{R}_{+}^{4}$ is positively invariant for system (1.2), it follows from Lemma 2.1 that $f^{t}(\Gamma \backslash D) \subset \Gamma \backslash D$, where $f^{t}(x)$ denotes the $t$ th iteration of $x$ under $f$. Moreover, employing Theorem 2.1 in [21] and Lemma 2.1, we know that there exists a global attractor $X$ in $\Gamma$.

Let $M=(0,0,0,0)$ be the maximal compact invariant set in $X$, and $\Gamma \backslash M$ is positively invariant. In order to prove if $r>1$, system (1.2) is uniformly persistent, which is equivalent to saying that $M$ is a uniform repeller. 
In the following, we will construct a continuous function $Q: \mathbb{R}_{+}^{4} \rightarrow \mathbb{R}_{+}$satisfying the conditions:

(1) $Q(x)=0$ for $x \in M$;

(2) there exists a neighborhood $U$ of $M$ such that $\forall x \in U \backslash M, \exists n>0$, such that $Q\left(f^{n}(x)\right)>Q(x)$.

Since $B(0)$ is non-negative and irreducible, it has a dominant eigenvalue $r>1$, which has a corresponding positive left eigenvector $\theta>0$,

$$
\theta^{T} B(0)=r \theta^{T} .
$$

Let $r^{*} \in(1, r)$ such that $\theta^{T} B(0)>r^{*} \theta^{T}$. Furthermore, there exists a neighbourhood $U$ of $M$ by the continuity of $B(x)$ such that

$$
\theta^{T} B(x)>r^{*} \theta^{T} .
$$

Define $Q: \mathbb{R}_{+}^{4} \rightarrow \mathbb{R}_{+}$as follows:

$$
Q(x)=\theta^{T} x .
$$

Then $Q(x)=0$ for $x \in U$ iff $x \in M$, and positive elsewhere in $U$. Moreover,

$$
Q(f(x))=\theta^{T} B(x) x>r^{*} \theta^{T} x>\theta^{T} x=Q(x), \quad \forall x \in U \backslash M .
$$

Hence, (1.2) is uniformly persistent. This implies that there exists a positive number $\rho \in$ $\mathbb{R}_{+}^{4}$ such that, for every solution $(E(t), L(t), P(t), A(t))$, we have

$$
\liminf _{t \rightarrow \infty}(E(t), L(t), P(t), A(t)) \geq \rho>0
$$

for all non-zero orbits in $\mathbb{R}_{+}^{4}$. This completes the proof.

In the following, we describe the situation when all translating rates equal one. Namely, $\gamma_{1}=\gamma_{2}=\gamma_{3}=1$. That is, we include a term that describes the survivorship of population from generation to generation. To proceed further, we review the relevant existing results on the $k+1$-order nonlinear difference equation before we formulate the problem that we subsequently study.

$$
x_{n+1}=F\left(x_{n}, x_{n-1}, \ldots, x_{n-k}\right), \quad n=0,1,2, \ldots
$$

where $F \in C\left(I^{k+1}, \mathbb{R}\right)$ and $I$ is an open interval of $\mathbb{R}$.

Lemma 2.2 Let $x^{*} \in I$ be an equilibrium of (2.7). Suppose that $F$ satisfies the following two conditions:

(1) $F$ is non-decreasing in each of its arguments;

(2) F satisfies $\left(u-x^{*}\right)[F(u, u, \ldots, u)-u]<0$ for all $u \in I \backslash x^{*}$.

Then equilibrium point $x^{*}$ is a global attractor of all solutions of equation (2.7). 
The analysis given below focuses on the case of the following system of difference equations:

$$
\left\{\begin{array}{l}
E(t+1)=b A(t), \\
L(t+1)=s_{1}(E(t)) E(t), \\
P(t+1)=s_{2}(L(t)) L(t), \\
A(t+1)=s_{3} P(t)+s_{4}(A(t)) A(t) .
\end{array}\right.
$$

We first observe that (2.8) has a trivial steady state $E_{0}$. It is easily seen that a nontrivial constant equilibrium $(\bar{E}, \bar{L}, \bar{P}, \bar{A})$ must be an equilibrium that has all positive components. The A-component of a nontrivial steady state must satisfy

$$
1=b s_{3} s_{2}\left(s_{1}(b \bar{A}) b \bar{A}\right) s_{1}(b \bar{A})+s_{4}(\bar{A}) .
$$

Consequently, equation (2.8) has a coexistence steady state $E^{*}=(\bar{E}, \bar{L}, \bar{P}, \bar{A})$, where all stages have positive density if and only if

$$
\frac{b s_{3} a_{1} a_{2}}{1-a_{4}}>1
$$

where $\bar{E}=b \bar{A}, \bar{L}=s_{1}(b \bar{A}) b \bar{A}, \bar{P}=s_{2}\left(s_{1}(b \bar{A}) b \bar{A}\right) s_{1}(b \bar{A}) b \bar{A}$. The interior steady state is unique whenever it exists. Specially, if we consider the inhibition of larvae density on the egg hatching, namely $s_{1}=s_{1}(L)$, then system (2.8) is the same as (1.1) in [12]. Consequently, we can use the stability information about the steady states to understand the asymptotic dynamics of our model.

Theorem 2.3 If $\Re_{0}(1,1,1)=\frac{b_{3} a_{1} a_{2}}{1-a_{4}}>1$, then (2.8) has a unique equilibrium $E^{*}=$ $(\bar{E}, \bar{L}, \bar{P}, \bar{A})$ which is globally asymptotically stable in the interior of $\mathbb{R}_{+}^{4}$.

Proof Since $\Re_{0}(1,1,1)>1$, it is clear that $E^{*}$ exists by the above analysis. We now turn our attention to equilibrium point $E^{*}$. First, we prove that $E^{*}$ is locally asymptotically stable. To calculate the local stability of $E^{*}$, the linearization of (2.8) about $E^{*}$ yields the Jacobian matrix

$$
J\left(E^{*}\right)=\left(\begin{array}{cccc}
0 & 0 & 0 & b \\
J_{21} & 0 & 0 & 0 \\
0 & J_{32} & 0 & 0 \\
0 & 0 & s_{3} & J_{44}
\end{array}\right),
$$

where $J_{21}=s_{1}^{\prime}(\bar{E}) \bar{E}+s_{1}(\bar{E})>0, J_{32}=s_{2}^{\prime}(\bar{L}) \bar{L}+s_{2}(\bar{L})>0$, and $J_{44}=s_{4}^{\prime}(\bar{A}) \bar{A}+s_{4}(\bar{A})>0$. Furthermore, at equilibrium $E^{*}$, the characteristic equation for the corresponding linearized model of (2.8) is

$$
f(\lambda)=\lambda^{4}-J_{44} \lambda^{3}-b s_{3} J_{21} J_{32}=0
$$


To show local asymptotic stability of $E^{*}$, we need to show that the following inequalities hold:

(i) $f(1)>0$,

(ii) $f(-1)>0$,

(iii) $\left|-b s_{3} J_{21} J_{32}\right|<1$,

(iv) $\left|1-b^{2} s_{3}^{2} J_{21}^{2} J_{32}^{2}\right|>\left|-b s_{3} J_{21} J_{32} J_{44}\right|$,

(v) $\left|\left(1-b^{2} s_{3}^{2} J_{21}^{2} J_{32}^{2}\right)^{2}-b^{2} s_{3}^{2} J_{21}^{2} J_{32}^{2} J_{44}^{2}\right|>\left|-b s_{3} J_{21} J_{32} J_{44}^{2}\right|$.

Since $\bar{A}$ satisfies (2.9), $\bar{E}=b \bar{A}, \bar{L}=s_{1}(\bar{E}) \bar{E}$, and $\bar{P}=s_{2}(\bar{L}) \bar{L}$, we have by (2.9) that

$$
1=b s_{3} s_{1}(\bar{E}) s_{2}(\bar{L})+s_{4}(\bar{A}) .
$$

Substituting the above expression of 1 into $f(1)$, and with the assumptions of $\left(H_{1}\right)$, we have

$$
\begin{aligned}
f(1) & =1-J_{44}-b s_{3} J_{21} J_{32} \\
& =1-\left(s_{4}^{\prime}(\bar{A}) \bar{A}+s_{4}(\bar{A})\right)-b s_{3}\left(s_{1}^{\prime}(\bar{E}) \bar{E}+s_{1}(\bar{E})\right)\left(s_{2}^{\prime}(\bar{L}) \bar{L}+s_{2}(\bar{L})\right) \\
& =-s_{4}^{\prime}(\bar{A}) \bar{A}-b s_{3} s_{2}(\bar{L}) s_{1}^{\prime}(\bar{E}) \bar{E}-b s_{3} s_{2}^{\prime}(\bar{L}) \bar{L}\left(s_{1}^{\prime}(\bar{E}) \bar{E}+s_{1}(\bar{E})\right)>0 .
\end{aligned}
$$

It is also clear that

$$
f(-1)=1+J_{44}-b s_{3} J_{21} J_{32}>2 J_{44}>0,
$$

as $J_{44}>0$. Next, we prove the third inequality

$$
\left|-b s_{3} J_{21} J_{32}\right|<1 \text {. }
$$

Note that the term inside the absolute value on the left-hand side of equation (2.12) is negative, yielding

$$
\begin{aligned}
\left|-b s_{3} J_{21} J_{32}\right| & =b s_{3} J_{21} J_{32}=b s_{3}\left(s_{1}^{\prime}(\bar{E}) \bar{E}+s_{1}(\bar{E})\right)\left(s_{2}^{\prime}(\bar{L}) \bar{L}+s_{2}(\bar{L})\right) \\
& <b s_{3} s_{1}(\bar{E}) s_{2}(\bar{L})<b s_{3} s_{1}(\bar{E}) s_{2}(\bar{L})+s_{4}(\bar{A})=1 .
\end{aligned}
$$

Because of equation (2.12) and $b, s_{3}, J_{21}, J_{32}, J_{44}>0$, condition (iv) becomes

$$
1-b^{2} s_{3}^{2} J_{21}^{2} J_{32}^{2}-b s_{3} J_{21} J_{32} J_{44}>0 .
$$

Since (i) has been satisfied, then

$$
\begin{aligned}
1-b^{2} s_{3}^{2} J_{21}^{2} J_{32}^{2}-b s_{3} J_{21} J_{32} J_{44} & =1-b s_{3} J_{21} J_{32}\left(b s_{3} J_{21} J_{32}+J_{44}\right), \\
& =1+b s_{3} J_{21} J_{32}(f(1)-1) \\
& =1-b s_{3} J_{21} J_{32}+b s_{3} J_{21} J_{32} f(1)>0 .
\end{aligned}
$$


At last, we proceed to verifying the last inequality (v). For convenience, we denote $m=$ $-b s_{3} J_{21} J_{32}, n=-J_{44}$. Then (v) is equal to

$$
\begin{aligned}
\mid( & \left.-b^{2} s_{3}^{2} J_{21}^{2} J_{32}^{2}\right)^{2}-b^{2} s_{3}^{2} J_{21}^{2} J_{32}^{2} J_{44}^{2}|-|-b s_{3} J_{21} J_{32} J_{44}^{2} \mid \\
\quad= & \left(1-m^{2}\right)^{2}-n^{2} m^{2}+n^{2} m \\
\quad & =(1-m)\left((1+m)^{2}(1-m)+n^{2} m\right) .
\end{aligned}
$$

Since $-1<m<0, n<0$, we have $1-m>0$. Then (v) is satisfied if $(1+m)^{2}(1-m)+n^{2} m>0$. However, by $f(1)=1+m+n>0$, we obtain

$$
(1+m)^{2}(1-m)+n^{2} m>n^{2}(1-m)+n^{2} m=n^{2}>0 .
$$

This implies that $E^{*}$ is locally asymptotically stable.

In what follows, we show the convergence of equilibrium $E^{*}$. Notice that (2.8) can be converted into the following scalar difference equation:

$$
A(t+4)=s_{3} s_{2}\left(s_{1}(b A(t)) b A(t)\right) s_{1}(b A(t)) b A(t)+s_{4}(A(t+3)) A(t+3) .
$$

Since $b s_{3} a_{1} a_{2}+a_{4}>1,(2.13)$ admits a unique positive steady state $\bar{A}$. It is sufficient to prove that $\bar{A}$ is globally attracting for $(2.13)$ in $(0, \infty)$.

Let

$$
g(E, L, P, A)=s_{3} s_{2}\left(s_{1}(b E) b E\right) s_{1}(b E) b E+s_{4}(A) A,
$$

we obtain

$$
\begin{aligned}
& \frac{\partial g}{\partial E}=s_{3}\left[s_{2}\left(s_{1}(b E) b E\right) s_{1}(b E) b E+s_{2}^{\prime}\left(s_{1}(b E) b E\right) s_{1}(b E) b E\right]\left[s_{1}^{\prime}(b E) b E+s_{1}(b E)\right]>0, \\
& \frac{\partial g}{\partial L}=\frac{\partial g}{\partial P}=0 \\
& \frac{\partial g}{\partial A}=s_{4}^{\prime}(A) A+s_{4}(A)>0
\end{aligned}
$$

for all $E, L, P, A>0$. Moreover,

$$
\begin{aligned}
(A & -\bar{A})[g(A, A, A, A)-A] \\
& =(A-\bar{A})\left[s_{3} s_{2}\left(s_{1}(b A) b A\right) s_{1}(b A) b A+s_{4}(A) A-A\right] \\
& =(A-\bar{A})\left[s_{3} s_{2}\left(s_{1}(b A) b A\right) s_{1}(b A) b+s_{4}(A)-1\right] A<0
\end{aligned}
$$

for $A>0$ and $A \neq \bar{A}$. Hence $\bar{A}$ is globally attracting for (2.13) in the interior of $\mathbb{R}_{+}^{4}$. By using Lemma 2.2, equilibrium $E^{*}$ is globally attracting for (2.8). This concludes the proof of this theorem.

Furthermore, we also know that system (2.8) is uniform in the parameters for a perturbation around $\gamma_{1}=\gamma_{2}=\gamma_{3}=1$. 
Theorem 2.4 If $\Re_{0}(1,1,1)=\frac{b_{3} a_{1} a_{2}}{1-a_{4}}>1$, then the extinction equilibrium for system (2.8) is a repeller, uniform in the parameter $\gamma=\left(\gamma_{1}, \gamma_{2}, \gamma_{3}\right)$ near $(1,1,1)$. That is, there is an open neighbourhood $U_{0}$ of 0 in $\mathbb{R}_{+}^{4}$, and positive constants $c_{i} \in(0,1), i=1,2,3$, such that, for every $x(0) \neq 0$ and every $\gamma \in\left[c_{1}, 1\right] \times\left[c_{2}, 1\right] \times\left[c_{3}, 1\right]$, there is some $N(x(0), \gamma) \geq 0$ such that

$$
f^{n}(x(t)) \notin U_{0}, \quad \forall n \geq N,
$$

where $x(t)=(E(t), L(t), P(t), A(t)), f(x(t))$ denotes the right-hand side of system (2.8), and $f^{n}(x)$ denotes the nth iteration of $x$ under $f$.

Proof The proof is similar to that of Ackleh ([19], Lemma 5) with some minor modifications, so we omit it here.

\section{Seasonal breeding}

In this section, we assume that breeding of the mosquito population is seasonal. Furthermore, to make the model mathematically tractable, we only consider a case for (2.8) with $b=b(t)$. That is to say, all survived individuals move into the subsequent stage within one time step. Then the model equations become

$$
\left\{\begin{array}{l}
E(t+1)=b(t) A(t), \\
L(t+1)=s_{1}(E(t)) E(t), \\
P(t+1)=s_{2}(L(t)) L(t), \\
A(t+1)=s_{3} P(t)+s_{4}(A(t)) A(t) .
\end{array}\right.
$$

We know that there are times when the environment is suitable for mosquitoes to lay, and to escape harsh environment, the adults do not lay eggs or eggs do not hatch. All their life processes drop to a very low level. Additionally, we assume in model (3.1) that the function $b(t)$ is periodic with period 2. Specifically, we let $b(0)=\widehat{b}, b(1)=0, b(2)=$ $\widehat{b}, b(3)=0, \ldots$ In other words, if the time unit is half a day and a full cycle consists of one day, the mosquito population gives birth only half the day, while in the other half there is no birth. Let $(E(0), L(0), P(0), A(0)) \in \mathbb{R}_{+}^{4} \backslash\{(0,0,0,0)\}$ be given. It is easy to see that $(E(t), L(t), P(t), A(t)) \in \mathbb{R}_{+}^{4} \backslash\{(0,0,0,0)\}$ for $t>0$. Moreover, from system (3.1), we get

$$
\begin{aligned}
& E(1)=b(0) A(0)=\widehat{b} A(0), \quad L(1)=s_{1}(E(0)) E(0), \\
& P(1)=s_{2}(L(0)) L(0), \quad A(1)=s_{3} P(0)+s_{4}(A(0)) A(0) .
\end{aligned}
$$

Hence, if $(E(0), L(0), P(0), A(0))$ is a part of two-cycle, with a little algebra one can show that $E(2)=b(1) A(1)=0, L(2)=s_{1}(\widehat{b} A(0)) \widehat{b} A(0), P(2)=s_{2}(L(1)) L(1)=0$, and

$$
A(2)=s_{3} s_{2}\left(s_{1}(\widehat{b} A(0)) \widehat{b} A(0)\right) s_{1}(\widehat{b} A(0)) \widehat{b} A(0)+s_{4}\left(s_{4}(A(0)) A(0)\right) s_{4}(A(0)) A(0) .
$$

If $A(0) \neq 0$, then $A(0)$ must satisfy

$$
1=s_{3} s_{2}\left(s_{1}(\widehat{b} A(0)) \widehat{b} A(0)\right) s_{1}(\widehat{b} A(0)) \widehat{b}+s_{4}\left(s_{4}(A(0)) A(0)\right) s_{4}(A(0)) .
$$


Let

$$
H(A)=s_{3} s_{2}\left(s_{1}(\widehat{b} A) \widehat{b} A\right) s_{1}(\widehat{b} A) \widehat{b}+s_{4}\left(s_{4}(A) A\right) s_{4}(A) .
$$

It follows that

$$
\begin{aligned}
H^{\prime}(A)= & \widehat{b} s_{3}\left[s_{2}^{\prime}\left(s_{1}(\widehat{b} A) \widehat{b} A\right) s_{1}(\widehat{b} A)\left(s_{1}(\widehat{b} A) \widehat{b} A\right)^{\prime}+s_{2}\left(s_{1}(\widehat{b} A) \widehat{b} A\right) s_{1}^{\prime}(\widehat{b} A) \widehat{b}\right] \\
& +s_{4}^{\prime}\left(s_{4}(A) A\right) s_{4}(A)\left(s_{4}(A) A\right)^{\prime}+s_{4}\left(s_{4}(A) A\right) s_{4}^{\prime}(A)
\end{aligned}
$$

for all $A>0$ and $s_{i}$ satisfies $(H 1)$ for $i=1,2,4$. Moreover, using similar calculations as before, one can show that $H(0)=\widehat{b} a_{1} a_{2} s_{3}+a_{4}^{2}, \lim _{A \rightarrow \infty} H(A)=0$, and $H^{\prime}(A)<0$. Denote $A(0)=A^{*}$, and thus, we conclude that (3.2) has a positive solution $A^{*}$ if and only if

$$
\widehat{b} a_{1} a_{2} s_{3}+a_{4}^{2}>1
$$

which is equivalent to

$$
\widehat{\Re}_{0}:=\frac{\widehat{b} a_{1} a_{2} s_{3}}{1-a_{4}^{2}}>1 \text {, }
$$

where $\widehat{\Re}_{0}$ is the inherent net reproductive number for the seasonal mosquito population. Let $L(0)=L^{*}$, therefore (3.1) has a unique two-cycle

$$
\left\{\left(0, L^{*}, 0, A^{*}\right),\left(\widehat{b} A^{*}, 0, s_{2}\left(L^{*}\right) L^{*}, s_{4}\left(A^{*}\right) A^{*}\right)\right\},
$$

if and only if (3.4) holds, where $A^{*}$ satisfies

$$
H\left(A^{*}\right)=s_{3} s_{2}\left(s_{1}\left(\widehat{b} A^{*}\right) \widehat{b} A^{*}\right) s_{1}\left(\widehat{b} A^{*}\right) \widehat{b}+s_{4}\left(s_{4}\left(A^{*}\right) A^{*}\right) s_{4}\left(A^{*}\right)=1
$$

and

$$
A^{*}=s_{3} s_{2}\left(L^{*}\right) L^{*}+s_{4}\left(s_{4}\left(A^{*}\right) A^{*}\right) s_{4}\left(A^{*}\right) A^{*} .
$$

Here, it is worth noting that system (3.1) is a periodic system. In the next theorem, we summarize the main stability results for linear non-autonomous system (3.1). To facilitate the proof of the theorem, we first establish a stability result that is of independent interest, following the analysis of Saber Elaydi [22].

For the autonomous (time-independent) linear system $x(n+1)=B x(n)$, the eigenvalues of $B$ determine the stability properties of the system. But for a periodic system $x(n+1)=B(n) x(n)$, the eigenvalues of $B(n)$ do not play any role in the determination of the stability properties of the system. Instead, the Floquet multipliers of $B(n)$ determine those properties. Considering the following periodic system:

$$
x(n+1)=B(n) x(n), \quad B(n+N)=B(n),
$$

we now give a simple but powerful criterion for asymptotic stability of system (3.6). 
Lemma 3.1 The steady state solution of equation (3.6) is asymptotically stable if and only if each eigenvalue of $C=B(N-1) B(N-2) \cdots B(0)$ has modulus less than 1.

Theorem 3.1 For $t=0,1,2, \ldots$, and $b(2 t)=\widehat{b}>0, b(2 t+1)=0$. Assume that $(H 1)$ holds. Then the following statements hold:

(i) If $\widehat{\Re}_{0}<1$, then $\widehat{E}_{0}=(0,0,0,0)$ is globally asymptotically stable.

(ii) If $\widehat{\Re}_{0}>1$, then there exists a unique nontrivial two-cycle for system (3.1) which is globally asymptotically stable.

Proof We divide the proof into the two cases.

First, we start by proving (i). By using Lemma 3.1, since system (3.1) is periodic with period two, the local stability of $\widehat{E}_{0}$ can be determined by establishing the eigenvalues of the projection matrix over a full cycle

$$
\left(\begin{array}{cccc}
0 & 0 & 0 & \widehat{b} \\
a_{1} & 0 & 0 & 0 \\
0 & a_{2} & 0 & 0 \\
0 & 0 & s_{3} & a_{4}
\end{array}\right)\left(\begin{array}{cccc}
0 & 0 & 0 & 0 \\
a_{1} & 0 & 0 & 0 \\
0 & a_{2} & 0 & 0 \\
0 & 0 & s_{3} & a_{4}
\end{array}\right)=\left(\begin{array}{cccc}
0 & 0 & \widehat{b} s_{3} & \widehat{b} a_{4} \\
0 & 0 & 0 & 0 \\
a_{1} a_{2} & 0 & 0 & 0 \\
0 & s_{3} a_{2} & s_{3} a_{4} & a_{4}^{2}
\end{array}\right) .
$$

Denote the resulting product matrix by $A_{0}$, then the characteristic polynomial associated with this matrix is given by

$$
g(\lambda)=\lambda^{2}\left(\lambda^{2}-a_{4}^{2} \lambda-\widehat{b} a_{1} a_{2} s_{3}\right)
$$

Then the eigenvalues of $A_{0}$ are 0 and the roots of equation

$$
\lambda^{2}-a_{4}^{2} \lambda-\widehat{b} a_{1} a_{2} s_{3}=0 .
$$

We conclude from [22] that the roots of (3.7) lie inside the unit disk if and only if

$$
1+a_{4}^{2}-\widehat{b} a_{1} a_{2} s_{3}>0, \quad 1-a_{4}^{2}-\widehat{b} a_{1} a_{2} s_{3}>0, \quad 1+\widehat{b} a_{1} a_{2} s_{3}>0,
$$

or, equivalently,

$$
\left|a_{4}^{2}\right|<1-\widehat{b} a_{1} a_{2} s_{3}<2 \text {. }
$$

If condition (3.8) holds, then $\widehat{E}_{0}$ is locally asymptotically stable. However, (3.8) can reduce to $a_{4}^{2}<1-\widehat{b} a_{1} a_{2} s_{3}<2$ as $a_{4}>0$. Note that $\widehat{b}>0, s_{3}>0$, and $a_{1}, a_{2}>0$ under assumption $\left(H_{1}\right)$, we know $1-\widehat{b} a_{1} a_{2} s_{3}<2$ is trivially true. Furthermore, the left-hand side of inequality is satisfied by $\widehat{\Re}_{0}<1$. Then (3.8) holds. Therefore, $\widehat{E}_{0}$ is locally asymptotically stable.

The next step is to establish the global attractivity of $\widehat{E}_{0}$ by virtue of a simple comparison method. From system (3.1), we see that $E(2 t+1)=\widehat{b} A(2 t), E(2 t+2)=0, L(2 t+1)=0$, $P(2 t+2)=0$, which implies that

$$
\begin{aligned}
& P(2 t+1) \leq a_{2} L(2 t), \quad A(2 t+1) \leq a_{4} A(2 t), \quad L(2 t+2) \leq a_{1} E(2 t+1), \\
& A(2 t+2)=s_{3} P(2 t+1)+s_{4}(A(2 t+1)) A(2 t+1),
\end{aligned}
$$


i.e.,

$$
L(2 t+2) \leq a_{1} \widehat{b} A(2 t), \quad A(2 t+2) \leq s_{3} a_{2} L(2 t)+a_{4}^{2} A(2 t)
$$

for $t \geq 1$. Letting $n+i=2(t+i)$ for $i \geq 0$, consider the following linear system of difference equations:

$$
M(n+1)=a_{1} \widehat{b} N(n), \quad N(n+1)=s_{3} a_{2} M(n)+a_{4}^{2} N(n),
$$

with $M(1)=L(2), N(1)=A(2)$.

$$
\left(\begin{array}{c}
M(n+1) \\
N(n+1)
\end{array}\right)=\left(\begin{array}{cc}
0 & \widehat{b} a_{1} \\
s_{3} a_{2} & a_{4}^{2}
\end{array}\right)\left(\begin{array}{l}
M(n) \\
N(n)
\end{array}\right) .
$$

From this, we get that the eigenvalues of the above coefficient matrix satisfy

$$
\lambda^{2}-a_{4}^{2} \lambda-\widehat{b} a_{1} a_{2} s_{3}=0 .
$$

Similar to the above analysis, all the eigenvalues have modulus less than 1 , and using a simple comparison method, we have $\lim _{n \rightarrow \infty} M(n)=\lim _{n \rightarrow \infty} N(n)=0$. Thus, $\lim _{n \rightarrow \infty} L(2 t+$ 2) $=\lim _{n \rightarrow \infty} A(2 t+2)=0$ and, as a result, $\lim _{n \rightarrow \infty} E(2 t+1) \leq \widehat{b} \lim _{n \rightarrow \infty} A(2 t)=0$, $\lim _{n \rightarrow \infty} P(2 t+1) \leq a_{2} \lim _{n \rightarrow \infty} L(2 t)=0$, and $\lim _{n \rightarrow \infty} A(2 t+1) \leq a_{4} \lim _{n \rightarrow \infty} A(2 t)=0$. Consequently, we know that $\widehat{E}_{0}$ is globally attracting of system (3.1). Therefore, $\widehat{E}_{0}$ is globally asymptotically stable.

From the above analysis, we have known that (3.1) has a unique two-cycle

$$
\left\{\left(0, L^{*}, 0, A^{*}\right),\left(\widehat{b} A^{*}, 0, s_{2}\left(L^{*}\right) L^{*}, s_{4}\left(A^{*}\right) A^{*}\right)\right\},
$$

if and only if $\widehat{\Re}_{0}>1$ holds, and

$$
L^{*}=s_{1}\left(\widehat{b} A^{*}\right) \widehat{b} A^{*} .
$$

In the following, we show that the two-cycle is globally asymptotically stable.

To prove (ii), we first prove that the two-cycle is locally asymptotically stable. Recall that its stability depends on the eigenvalues of the product of the matrices

$$
\left(\begin{array}{cccc}
0 & 0 & 0 & \widehat{b} \\
a_{1} & 0 & 0 & 0 \\
0 & a_{32} & 0 & 0 \\
0 & 0 & s_{3} & a_{44}
\end{array}\right)\left(\begin{array}{cccc}
0 & 0 & 0 & 0 \\
C & 0 & 0 & 0 \\
0 & a_{2} & 0 & 0 \\
0 & 0 & s_{3} & B
\end{array}\right)=\left(\begin{array}{cccc}
0 & 0 & \widehat{b} s_{3} & \widehat{b} B \\
0 & 0 & 0 & 0 \\
a_{32} C & 0 & 0 & 0 \\
0 & s_{3} a_{2} & s_{3} a_{44} & B a_{44}
\end{array}\right),
$$

where

$$
\begin{aligned}
& a_{32}=s_{2}^{\prime}\left(L^{*}\right) L^{*}+s_{2}\left(L^{*}\right)>0, \\
& a_{44}=s_{4}^{\prime}\left(A^{*}\right) A^{*}+s_{2}\left(A^{*}\right)>0,
\end{aligned}
$$




$$
\begin{aligned}
& B=s_{4}^{\prime}\left(s_{4}\left(A^{*}\right) A^{*}\right) s_{4}\left(A^{*}\right) A^{*}+s_{4}\left(s_{4}\left(A^{*}\right) A^{*}\right)>0, \\
& C=s_{1}^{\prime}\left(\widehat{b} A^{*}\right) \widehat{b} A^{*}+s_{1}\left(\widehat{b} A^{*}\right)>0 .
\end{aligned}
$$

Denote the resulting product matrix by $A_{1}$. Then the characteristic polynomial associated with this matrix is given by

$$
p(\lambda)=\lambda^{2}\left(\lambda^{2}-B a_{44} \lambda-\widehat{b} s_{3} a_{32} C\right) .
$$

Then the eigenvalues of $A_{1}$ are 0 and the roots of equation $\lambda^{2}-B a_{44} \lambda-\widehat{b s}_{3} a_{32} C=0$. In order to show that the two-cycle is locally asymptotically stable, it is necessary and sufficient that

$$
\left|-B a_{44}\right|<1-\widehat{b}_{3} a_{32} C<2
$$

which reduces to $B a_{44}<1-\widehat{b} s_{3} a_{32} C<2$ as $B, a_{44}>0$. Note that $\widehat{b}, s_{3}>0$ and $a_{32}, C>0$ under assumption $\left(H_{1}\right)$, we know $1-\widehat{b} s_{3} a_{32} C<2$ is always true. Therefore, we only need to prove

$$
1-\widehat{b} s_{3} a_{32} C-B a_{44}>0
$$

Replacing 1 by the right-hand side of equilibrium equation (3.5), we have

$$
\begin{aligned}
1-\widehat{b} s_{3} a_{32} C-B a_{44} \\
=1-\widehat{b} s_{3} s_{2}^{\prime}\left(L^{*}\right) L^{*} s_{1}^{\prime}\left(\widehat{b} A^{*}\right) \widehat{b} A^{*}-\widehat{b} s_{3} s_{2}^{\prime}\left(L^{*}\right) L^{*} s_{1}\left(\widehat{b} A^{*}\right)-\widehat{b} s_{3} s_{2}\left(L^{*}\right) s_{1}\left(\widehat{b} A^{*}\right) \\
\quad-\widehat{b} s_{3} s_{2}\left(L^{*}\right) s_{1}^{\prime}\left(\widehat{b} A^{*}\right) \widehat{b} A^{*}-s_{4}^{\prime}\left(s_{4}\left(A^{*}\right) A^{*}\right) s_{4}\left(A^{*}\right) A^{*} s_{4}^{\prime}\left(A^{*}\right) A^{*} \\
\quad-s_{4}^{\prime}\left(s_{4}\left(A^{*}\right) A^{*}\right) s_{4}\left(A^{*}\right) A^{*} s_{4}\left(A^{*}\right)-s_{4}\left(s_{4}\left(A^{*}\right) A^{*}\right) s_{4}^{\prime}\left(A^{*}\right) A^{*}-s_{4}\left(A^{*}\right) s_{4}\left(s_{4}\left(A^{*}\right) A^{*}\right) \\
=\widehat{b} s_{3} s_{2}\left(s_{1}\left(\widehat{b} A^{*}\right) \widehat{b} A^{*}\right) s_{1}\left(\widehat{b} A^{*}\right)-\widehat{b} s_{3} s_{2}\left(L^{*}\right)\left[s_{1}^{\prime}\left(\widehat{b} A^{*}\right) \widehat{b} A^{*}+s_{1}\left(\widehat{b} A^{*}\right)\right] \\
\quad-\widehat{b} s_{3} s_{2}^{\prime}\left(L^{*}\right) L^{*}\left[s_{1}^{\prime}\left(\widehat{b} A^{*}\right) \widehat{b} A^{*}+s_{1}\left(\widehat{b} A^{*}\right)\right] \\
\quad-s_{4}^{\prime}\left(s_{4}\left(A^{*}\right) A^{*}\right) s_{4}\left(A^{*}\right) A^{*}\left[s_{4}^{\prime}\left(A^{*}\right) A^{*}+s_{4}\left(A^{*}\right)\right] \\
\quad-s_{4}\left(s_{4}\left(A^{*}\right) A^{*}\right) s_{4}^{\prime}\left(A^{*}\right) A^{*} .
\end{aligned}
$$

Notice that the last three terms in the above expression are all positive, and the first two terms can be combined to yield a positive term $-\widehat{b} s_{3} s_{2}\left(L^{*}\right) s_{1}^{\prime}\left(\widehat{b} A^{*}\right) \widehat{b} A^{*}$ by (3.10). Therefore (3.11) holds and the two-cycle is locally asymptotically stable.

To finish the proof of (ii), it remains to show that the two-cycle is globally attracting in the interior of $\mathbb{R}_{+}^{4}$. The proof is similar to the proof of Sect. 2. Let $(E(0), L(0), P(0), A(0)) \in$ $\mathbb{R}_{+}^{4} \backslash\{(0,0,0,0)\}$ be given. Note that

$$
\begin{aligned}
& E(2 t+1)=b(2 t) A(2 t)=\widehat{b} A(2 t), \quad P(2 t+1)=s_{2}(L(2 t)) L(2 t), \\
& A(2 t+1)=s_{4}(A(2 t)) A(2 t)
\end{aligned}
$$


for $t \geq 1$. From this, we get

$$
\begin{aligned}
L(2 t+2) & =s_{1}(E(2 t+1)) E(2 t+1)=s_{1}(\widehat{b} A(2 t)) \widehat{b} A(2 t), \\
A(2 t+2) & =s_{3} P(2 t+1)+s_{4}(A(2 t+1)) A(2 t+1) \\
& =s_{3} s_{2}(L(2 t)) L(2 t)+s_{4}\left(s_{4}(A(2 t)) A(2 t)\right) s_{4}(A(2 t)) A(2 t) .
\end{aligned}
$$

Let $n+i=2(t+i)$ for $i \geq 0$, we obtain

$$
\begin{aligned}
G(n+1) & =s_{1}(\widehat{b} K(n)) \widehat{b} K(n), \\
K(n+1) & =s_{3} s_{2}(G(n)) G(n)+s_{4}\left(s_{4}(K(n)) K(n)\right) s_{4}(K(n)) K(n)
\end{aligned}
$$

for $n \geq 1$, which is equivalent to the following second-order scalar equation:

$$
\begin{aligned}
K(n+2)= & \left.s_{3} s_{2}\left(s_{1}(\widehat{b} K(n)) \widehat{b} K(n)\right) s_{1} \widehat{b} K(n)\right) \widehat{b} K(n) \\
& +s_{4}\left(s_{4}(K(n+1)) K(n+1)\right) s_{4}(K(n+1)) K(n+1) .
\end{aligned}
$$

Let the right-hand side of (3.14) be denoted by $q(x, y)$, i.e.,

$$
\left.q(x, y)=s_{3} s_{2}\left(s_{1}(\widehat{b} x) \widehat{b} x\right) s_{1}(\widehat{b} x) \widehat{b} x\right)+s_{4}\left(s_{4}(y) y\right) s_{4}(y) y .
$$

Then

$$
\frac{\partial q}{\partial x}=s_{3}\left[s_{2}^{\prime}\left(s_{1}(\widehat{b} x) \widehat{b} x\right) s_{1}(\widehat{b} x) \widehat{b} x+s_{2}\left(s_{1}(\widehat{b} x) \widehat{b} x\right)\right]\left(s_{1}(\widehat{b} x) \widehat{b} x\right)^{\prime}>0
$$

and

$$
\frac{\partial q}{\partial y}=\left[s_{4}^{\prime}\left(s_{4}(y) y\right) s_{4}(y) y+s_{4}\left(s_{4}(y) y\right)\right]\left(s_{4}(y) y\right)^{\prime}>0 .
$$

Furthermore, (3.14) admits a unique interior steady state $A^{*}$ since $\widehat{b} a_{1} a_{2} s_{3}+a_{4}^{2}>1$. It is easy to see that

$$
\left.\left(u-A^{*}\right)[q(u, u)-u]=\left(u-A^{*}\right)\left[s_{3} s_{2}\left(s_{1}(\widehat{b} u) \widehat{b} u\right) \widehat{b} u\right) \widehat{b} u+s_{4}\left(s_{4}(u) u\right) s_{4}(u) u-1\right] u<0 .
$$

Employing the results of Lemma 2.2, we have the following result, $\lim _{t \rightarrow \infty} L(2 t)=L^{*}$, $\lim _{t \rightarrow \infty} A(2 t)=A^{*}$. That is to say, the even subsequence $(E(2 t), L(2 t), P(2 t), A(2 t))$ of the solution converges to $\left(0, L^{*}, 0, A^{*}\right)$. Meanwhile, it follows from (3.12) and (3.13), we obtain $\lim _{t \rightarrow \infty} E(2 t+1)=\widehat{b} A^{*}, \lim _{t \rightarrow \infty} P(2 t+1)=s_{2}\left(L^{*}\right) L^{*}$, and $\lim _{t \rightarrow \infty} A(2 t+1)=s_{4}\left(A^{*}\right) A^{*}$, and thereby the odd subsequence $(E(2 t+1), L(2 t+1), P(2 t+1), A(2 t+1))$ converges to $\left.\widehat{b} A^{*}, 0, s_{2}\left(L^{*}\right) L^{*}, s_{4}\left(A^{*}\right) A^{*}\right)$. Since $(E(0), L(0), P(0), A(0)) \neq(0,0,0,0)$ is arbitrary, we get that the two-cycle solution is globally attracting in the interior of $\mathbb{R}_{+}^{4}$. Hence, the two-cycle is globally asymptotically stable.

Remark 3.1 For system (3.6), every local stability property of the zero solution implies the corresponding global stability property. 


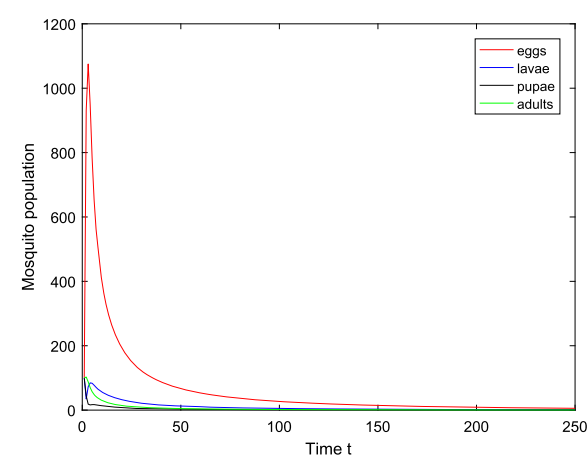

(a)

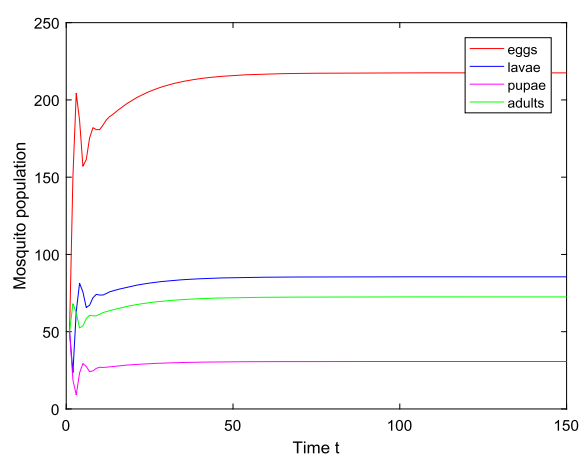

(b)

Figure 1 The red, blue, black, and green curves correspond to the eggs, larvae, pupae, adults classes, respectively. The survivorship functions are $s_{i}(x)=a_{i} k_{i} /\left(k_{i}+a_{i} x\right), i=1,2,4$, with parameter values $a_{1}=0.5$, $a_{2}=0.4, a_{4}=0.8, k_{1}=400, k_{2}=300, k_{4}=800, \gamma_{1}=0.3, \gamma_{2}=0.4, \gamma_{3}=0.5$, and $s_{3}=0.6$. The initial conditions are given by $E(0)=L(0)=P(0)=A(0)=50$. If $b=9$, the inherent net reproductive number $\Re_{0}=0.9370<1$, mosquito-free equilibrium $E_{0}$ is globally asymptotically stable. Solution approach $E_{0}$ as $t \rightarrow \infty$, shown in the left figure, where $\gamma_{1}=\gamma_{2}=\gamma_{3}=1$, with the same function forms and some parameters. As $b=3$, the inherent net reproductive number $\Re_{0}(1,1,1)=1.8>1, E_{1}=(30,70,82,210)$ is globally asymptotically stable. Solutions approach $E_{1}$, as $t \rightarrow \infty$, shown in the right figure

\section{Simulations, biological explanations, and discussions}

In order to interpret the conclusions from a quantitative perspective, the dynamics of the mosquito population by numerical simulations will be analyzed in the following. In this section, we use MATLAB technical computing software to find the numerical solutions of model (1.2) and analyze the effect of basic reproduction number $\Re_{0}$ and $\widehat{\Re}_{0}$.

With parameter values given in Fig. 1, the breeding of mosquito population is continuous, if $b=9$, the inherent net reproductive number $\Re_{0}=0.9370<1$ (see Fig. 1(a)). Thus, Theorem 2.1 indicates that mosquito-free equilibrium $E_{0}$ is globally asymptotically stable, and the mosquito population goes extinct. Meanwhile, this result shows that the mosquito population can be eliminated by controlling birth rate of adults. If $b=12$, the inherent net reproductive number $\Re_{0}=1.2493>1$. While we are only to show the persistence of the positive fixed point for model (1.2) with the Beverton-Holt form survival functions, the stability seems global.

Next, we analyze numerically the global dynamics of system (1.2) for $\gamma_{1}=\gamma_{2}=\gamma_{3}=1$.

Figure 1 (b) shows that if the inherent net reproductive number $\mathfrak{R}_{0}(1,1,1)=1.8>1$, the trivial fixed point becomes unstable, and there exists an interior steady state, $E_{1}=$ $(30,70,82,210)$, which is globally asymptotically stable. It means that the mosquito population exists despite the low birth rates of adults in this case.

Figure 2 indicates that for model (3.1), when the breeding of the mosquito population is seasonal with 2-cycle, i.e., $b(2 t)=\widehat{b}, b(2 t+1)=0$. As $\widehat{b}=2.5$, the inherent net reproductive number $\widehat{\Re}_{0}=0.8333<1, \widehat{E}_{0}$ is globally asymptotically stable and the mosquito population can be eliminated. As $\widehat{b}=4, \widehat{\Re}_{0}=1.3333>1$, a stable 2 -cycle appears which is globally asymptotically stable and the mosquito population can be established. Comparing the results with those above, we know that period 2 birth rates are not advantageous for low birth rates of the mosquito population. Furthermore, we remark that model (3.1) admits a periodic solution which illustrates our mathematical result of Theorem 3.1. 

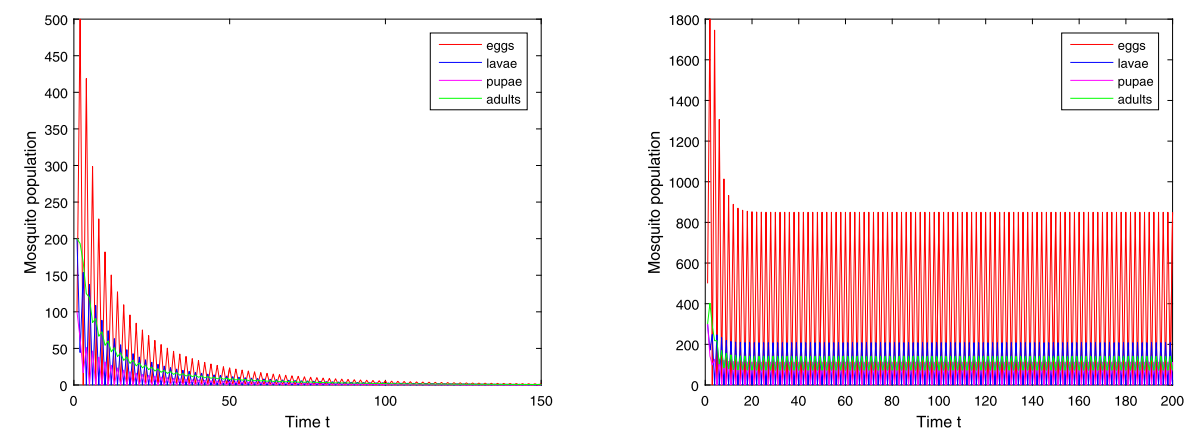

Figure 2 The function forms and some parameters are given in Fig. 1. When the breeding of the mosquito population is seasonal with 2 -cycle, $b(2 t)=\widehat{b}, b(2 t+1)=0$. As $\widehat{b}=2.5$, the inherent net reproductive number $\widehat{\Re}_{0}=0.8333<1, \widehat{E}_{0}$ is globally asymptotically stable. Solutions approach $\widehat{E}_{0}$ as $t \rightarrow \infty$, shown in the left figure. As $\widehat{b}=4, \widehat{\Re}_{0}=1.3333>1$, and a stable 2-cycle appears, which is globally asymptotically stable, shown in the right figure
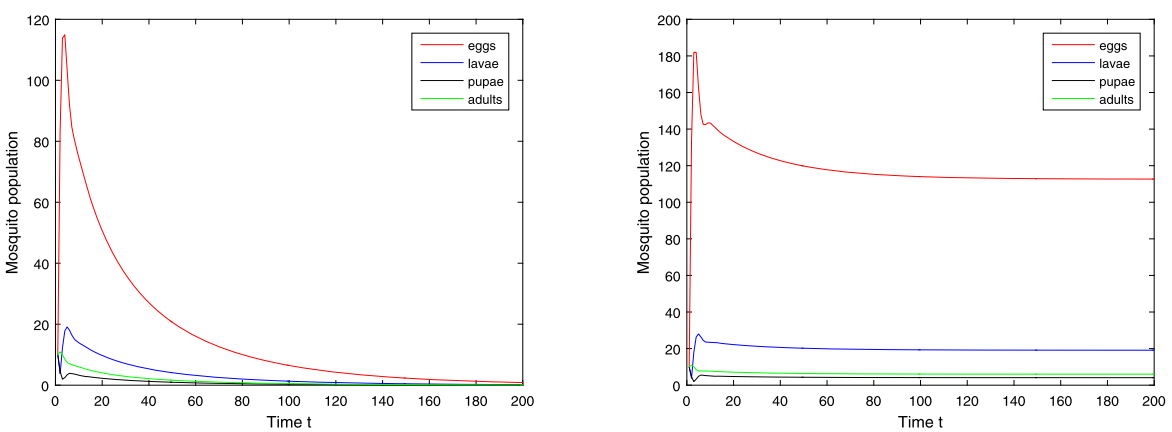

Figure 3 The functions $s_{1}(E)$ and $s_{4}(A)$ still have the Beverton-Holt form such that $s_{i}(x)=a_{i} k_{i} /\left(k_{i}+a_{i} x\right), i=1,4$, but $s_{2}(L)$ has the Ricker-type nonlinearity such that $s_{2}(L)=a_{2} e^{-L / k_{2}}$ and $E(0)=L(0)=P(0)=A(0)=10$, other parameters are given in Fig. 1. As $b=8$, the inherent net reproductive number $\Re_{0}=0.8329<1$, trivial fixed point $E_{0}$ is globally asymptotically stable. Solutions approach $E_{0}$ as $t \rightarrow \infty$, shown in the left figure. As $b=13$, $\Re_{0}=1.3534>1, E_{0}$ becomes unstable and $E_{1}=(4.29,5.71,20,114.2)$, which is globally asymptotically stable, shown in the right figure

With all parameter values given in Fig. $1, s_{2}(L)$ has the Ricker-type nonlinearity such that $s_{2}(L)=a_{2} e^{-L / k_{2}}$ and $E(0)=L(0)=P(0)=A(0)=10$. Figure 3 shows that for model (1.2), the breeding of the mosquito population is a constant. As $b=8$, the inherent net reproductive number $\Re_{0}=0.8329<1$, mosquito-free equilibrium $\widetilde{E}_{0}$ is globally asymptotically stable. As $b=13, \Re_{0}=1.3534>1, \widetilde{E}_{0}$ becomes unstable and $\widetilde{E}_{1}=(4.29,5.71,20,114.2)$, the system is globally asymptotically stable. We find that the solution curve is the same as in Fig. 1 . It means that the changing larval survival rate has little effect on the stability of equilibrium state.

To conclude, our numerical simulations demonstrate when the function $s_{i}(x)$ has the Ricker-type form such that $s_{i}(x)=a_{i} e^{-x / k_{i}}, i=1,2,4$, complex behavior emerges on the dynamic of the mosquito population. Here, we use the following set of parameters for the model of (1.2): $a_{1}=0.7, a_{2}=0.4, s_{3}=0.68, a_{4}=0.2, k_{1}=300, k_{2}=350, k_{4}=500, \gamma_{1}=$ $0.8, \gamma_{2}=0.8, \gamma_{3}=0.8$, and let $b$ vary, but keep all $\Re_{0}>1$ and $\Re_{0}$ increasing. The periodic oscillations appear as expected. The larger the $\Re_{0}$, the larger the amplitude of solution, for 
different values of $\Re_{0}$, the dynamical behavior of solutions changes. As $b=12, \Re_{0}=2.1391$. The positive fixed point is globally asymptotically stable. As $b=70, \Re_{0}=12.4780$. The solution appears to oscillate. As $b=100, \Re_{0}=17.8257$, and a cycle with period exists. As $b=200, \Re_{0}=35.6514$, and the system exhibits chaotic behavior. All populations shown in the figures are only eggs for clearer views.

Mosquito-borne diseases are a major public health concern because they cause substantial mortality and morbidity worldwide. An effective way to prevent these diseases is to control mosquitoes. Hence, it is crucial to study the population dynamics of mosquitoes and to devise effective and realistic methods for controlling mosquito population in communities. To build a theoretical framework, we developed and analysed discrete-time models for mosquitoes, including the four distinct stages with timedependent(independent) birth rates and nonlinear survivorship rates.

For model (1.2), we show that for continuous or seasonal breeding, extinction or coexistence depends on the inherent net reproductive number. First, continuous breeding is assumed and the asymptotic behavior of the resulting autonomous model is fully analyzed. It is shown that the unique interior equilibrium is uniformly persistent when the inherent net reproductive number is greater than one. Furthermore, if we assume the transition rate from the previous to the next state, i.e., $\gamma_{1}=\gamma_{2}=\gamma_{3}=1$, the unique interior equilibrium is globally asymptotically stable. However, when the inherent net reproductive is less than one, the mosquito population becomes extinct. In addition, we also show that the system is uniformly persistent in the parameters with a perturbation around $\gamma_{1}=\gamma_{2}=\gamma_{3}=1$. Then, we fully analyze the model with a seasonal breeding birth rate for the above special case. That is to say, all survived individuals move into the following stage within one time step, respectively, a seasonal breeding birth rate with period two is assumed. It is proved that for this non-autonomous model a period two solution is globally asymptotically stable when the inherent net reproductive number is greater than one, and when the inherent net reproductive number is less than one, the population becomes extinct. The outcomes rely on reducing the model to a higher-order scalar difference equation and applying the result on monotone scalar difference equations from [22].

Finally, numerical simulations are also provided to demonstrate these theoretical results. Moreover, in order to study the effects of survivorship functions which are strong non-linearities as opposed to weak non-linearities, we consider the dynamical properties for a case with the Ricker-type form survival functions in our model. In this case, the dynamics are complicated and the behaviour of the solutions is captured primarily through simulations. In Fig. 4, we consider Ricker-type survivorship functions with a constant birth, we use the following Ricker-type survivorship functions: $s_{i}(x)=a_{i} e^{-x / k_{i}}$ for $i=1,2,4$. The parameter values and initial conditions are set in Fig. 4 . We see that the Ricker-type survivorship functions result in much richer dynamics than those with the Beverton-Holt functions, including chaotic dynamics. We also plan to incorporate this investigation into the mosquito-borne disease. Mathematical analysis will be more challenging, but this is exactly what we need. Our objective is to find the best strategies for the releases of sterile mosquitoes to prevent and control the mosquito-borne diseases. 

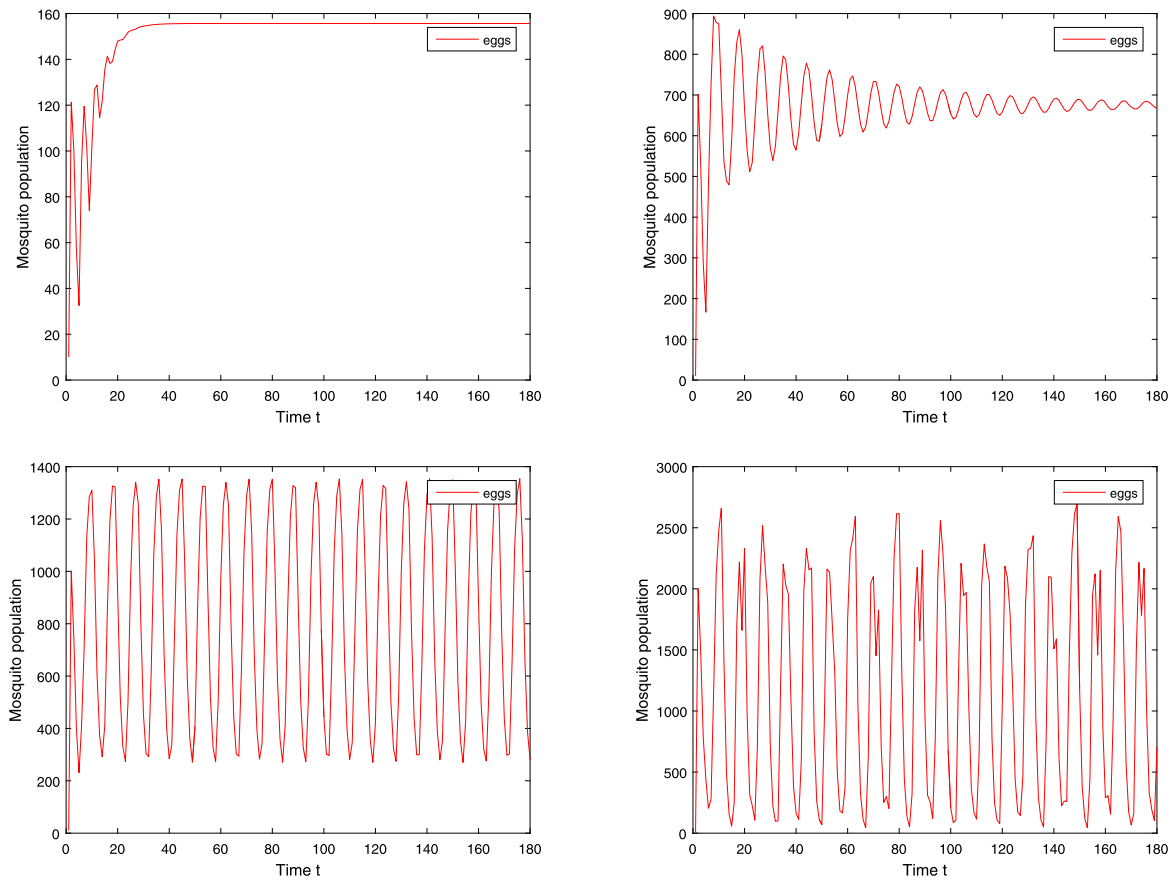

Figure 4 The function $s_{i}(x)$ has the Ricker-type form such that $s_{i}(x)=a_{i} e^{-x / k_{i}}, i=1,2,4$, with the parameters $a_{1}=0.7, a_{2}=0.4, s_{3}=0.68, a_{4}=0.2, k_{1}=300, k_{2}=350, k_{4}=500, r_{1}=0.8, r_{2}=0.8, r_{3}=0.8$, As $b=12$,

$\Re_{0}=2.1391$. The positive fixed point is globally asymptotically stable, as shown in the upper left figure. As $b=70, \Re_{0}=12.4780$. The solution appears to oscillate, as shown in the upper right figure. As $b=100$,

$\Re_{0}=17.8257$, and a cycle with period exists, as shown in the lower left figure. As $b=200, \Re_{0}=35.6514$, and the system exhibits chaotic behavior, as shown in the lower right figure. All populations shown in the figures are only eggs for clearer views

\section{Acknowledgements}

We are thankful to the editor and the anonymous reviewers for many valuable suggestions to improve this paper.

\section{Funding}

This work was supported by the National Natural Science Foundation of China (Nos. 11771104, 11871171), Scientific and Technological Innovation Programs of Higher Education Institutions in Shanxi (2019L0964). The authors, therefore, acknowledge with thanks NNSF, STIP for financial support.

\section{Availability of data and materials}

Data sharing not applicable to this article as no datasets were generated or analysed during the current study.

\section{Competing interests}

The authors declare that they have no competing interests.

\section{Authors' contributions}

All authors contributed equally to the manuscript and typed, read, and approved the final manuscript.

\section{Author details}

${ }^{1}$ School of Mathematics and Information Sciences, Guangzhou University, Guangzhou, P.R. China. ${ }^{2}$ Department of Mathematics, Luliang University, Luliang, P.R. China.

\section{Publisher's Note}

Springer Nature remains neutral with regard to jurisdictional claims in published maps and institutional affiliations.

Received: 26 June 2019 Accepted: 3 December 2019 Published online: 12 December 2019

\section{References}

1. Moulay, D., Aziz Alaoui, M.A., Cadivel, M.: The chikungunya disease: modelling, vector and transmission global dynamics. Math. Biosci. 229(1), 50-63 (2010) 
2. Balsacchino, F., Caputo, B., Chandre, F.: Control methods against invasive Aedes mosquitoes in Europe: a review. Pest Manag. Sci. 71(11), 1471-1485 (2015)

3. Faraji, A., Unlu, l.: The eye of the tiger, the thrill of the fight: effective larval and adult control measures against the Asian tiger mosquito, Aedes albopictus(diptera: culicidae), in North America. J. Med. Entomol. 53(5), 1029-1047 (2016)

4. Zheng, B., Yu, J., Xi, Z.: The annual abundance of dengue and Zika vector Aedes albopictus and its stubbornness to suppression. Ecol. Model. 387, 38-48 (2018)

5. Becker, N.: Mosquitoes and Their Control. Kluwer Academic, New York (2003)

6. Chitnis, N., Hyman, J.M., Cushing, J.M.: Determining important parameters in the spread of malaria through the sensitivity analysis of a mathematical model. Bull. Math. Biol. 70(5), 1272-1296 (2008)

7. Li, J.: New revised simple models for interactive wild and sterile mosquito population and their dynamics. J. Biol. Dyn. $11(2), 316-333(2017)$

8. Li, J.: Discrete-time models with mosquitoes carrying genetically-modified bacteria. Math. Biosci. 240(1), 35-44 (2012)

9. Traoré, B., Sangaré, B., Traoré, S.: A mathematical model of malaria transmission with structured vector population and seasonality. J. Appl. Math. 2017, Article ID 6754097 (2017)

10. Koutou, O., Traoré, B., Sangaré, B.: Mathematical modeling of malaria transmission global dynamics: taking into account the immature stages of the vectors. Adv. Differ. Equ. 2018, 220 (2018)

11. Lutambi, A.M., Penny, M.A., Smith, T.: Mathematical modelling of mosquito dispersal in a heterogeneous environment. Math. Biosci. 241(2), 198-216 (2013)

12. Lu, J., Li, J.: Dynamics of stage-structured discrete mosquito population models. J. Appl. Anal. Comput. 1(1), 53-67 (2011)

13. Tian, C., Ruan, S.: A free boundary problem for Aedes aegypti mosquito invasion. Appl. Math. Model. 46, 203-217 (2017)

14. Huang, M., Hu, L., Zheng, B.: Comparing the efficiency of Wolbachia driven Aedes mosquito suppression strategies. J. Appl. Anal. Comput. 9(1), 211-230 (2019)

15. Caswell, H.: Matrix Population Models. Sinauer, Sunderland (2001)

16. Cushing, J.M.: An Introduction to Structured Population Dynamics. Society for Industrial and Applied Mathematics, Philadelphia (1998)

17. Cushing, J.M., Yicang, Z.: The net reproductive value and stability in matrix population models. Nat. Resour. Model. 8(4), 297-333 (1994)

18. Ackleh, A.S., Jang, J.: A discrete two-stage population model: continuous versus seasonal reproduction. J. Differ. Equ. Appl. 13(4), 261-274 (2007)

19. Ackleh, A.S., Leenheer, P.D.: Discrete three-stage population model: persistence and global stability results. J. Biol. Dyn. 2(4), 415-427 (2008)

20. Ackleh, A.S., Chiquet, R.A.: The global dynamics of a discrete juvenile-adult model with continuous and seasonal reproduction. J. Biol. Dyn. 3(2-3), 101-115 (2009)

21. Hale, J.K., Waltman, P.: Persistence in infinite-dimensional systems. SIAM J. Math. Anal. 20(2), 388-395 (1989)

22. Elaydi, S.: An Introduction to Difference Equations, 3rd edn. Springer, New York (2005)

\section{Submit your manuscript to a SpringerOpen ${ }^{\circ}$ journal and benefit from:}

- Convenient online submission

- Rigorous peer review

- Open access: articles freely available online

- High visibility within the field

- Retaining the copyright to your article

Submit your next manuscript at $\boldsymbol{~ s p r i n g e r o p e n . c o m ~}$ 